\title{
Proteotranscriptomics assisted gene annotation and spatial proteomics of Bombyx mori BmN4 cell line
}

\author{
Michal Levin ${ }^{*}$ D, Marion Scheibe and Falk Butter
}

\begin{abstract}
Background: The process of identifying all coding regions in a genome is crucial for any study at the level of molecular biology, ranging from single-gene cloning to genome-wide measurements using RNA-seq or mass spectrometry. While satisfactory annotation has been made feasible for well-studied model organisms through great efforts of big consortia, for most systems this kind of data is either absent or not adequately precise.

Results: Combining in-depth transcriptome sequencing and high resolution mass spectrometry, we here use proteotranscriptomics to improve gene annotation of protein-coding genes in the Bombyx mori cell line BmN4 which is an increasingly used tool for the analysis of piRNA biogenesis and function. Using this approach we provide the exact coding sequence and evidence for more than 6200 genes on the protein level. Furthermore using spatial proteomics, we establish the subcellular localization of thousands of these proteins. We show that our approach outperforms current Bombyx mori annotation attempts in terms of accuracy and coverage.

Conclusions: We show that proteotranscriptomics is an efficient, cost-effective and accurate approach to improve previous annotations or generate new gene models. As this technique is based on de-novo transcriptome assembly, it provides the possibility to study any species also in the absence of genome sequence information for which proteogenomics would be impossible.
\end{abstract}

Keywords: Proteotranscriptomics, Mass spectrometry, Gene assembly, Gene annotation, Spatial proteomics

\section{Background}

Bombyx mori was the first lepidopteran species whose draft genome was published in 2004 [1, 2]. In 2008, a more accurate genome assembly was generated by combining the raw data of these initial efforts within an international collaboration [3], and the results are available at SilkDB (www.silkdb.org) and KAIKObase (sgp.dna.affrc. go.jp/index.html). However, for a large number of modern applications such as transcriptomic, epigenomic and proteomic studies, reverse genetic screens and genome editing tools such as TALEN and CRISPR/Cas9 the

\footnotetext{
* Correspondence: m.levin@imb.de; f.butter@imb.de Institute of Molecular Biology (IMB), Ackermannweg 4, 55128 Mainz, Germany
}

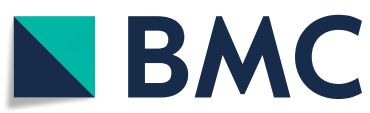

(c) The Author(s). 2020, corrected publication 2020. Open Access This article is licensed under a Creative Commons Attribution 4.0 International License, which permits use, sharing, adaptation, distribution and reproduction in any medium or format, as long as you give appropriate credit to the original author(s) and the source, provide a link to the Creative Commons licence, and indicate if changes were made. The images or other third party material in this article are included in the article's Creative Commons licence, unless indicated otherwise in a credit line to the material. If material is not included in the article's Creative Commons licence and your intended use is not permitted by statutory regulation or exceeds the permitted use, you will need to obtain permission directly from the copyright holder. To view a copy of this licence, visit http://creativecommons.org/ licenses/by/4.0/. The Creative Commons Public Domain Dedication waiver (http://creativecommons.org/publicdomain/zero/1. 0/) applies to the data made available in this article, unless otherwise stated in a credit line to the data. provided genome information is insufficient as this assembly contains numerous non-sequenced chromosome regions. Recently, parallel to our efforts to reannotate Bombyx mori using proteotranscriptomics, two new initiatives provided improved genome assemblies. These new assemblies have been made available as SilkBase [4] and SilkDB 3.0 [5] and include more genomic regions and gene predictions for 16,880 and 16,069 gene models, receptively. However, the provided gene models are still based on automated gene prediction using limited fulllength cDNA libraries, poly-A RNA-seq data and previous B. mori NCBI annotations. These predictions are made with a mixture of data from various commercial and noncommercial strains of Bombyx mori, thus may not represent the genomic sequence of a single strain or its derived 
cell line due to intraspecies genetic variation. Furthermore, only very few predicted Bombyx mori genes have evidence at the protein level (roughly 150 genes in UniProt UP000005204). Hence, an improved strain-specific gene annotation would likely improve global analyses.

Bombyx mori is similar to humans in terms of sensitivities to pathogens and comparable effects of drugs. The advantages for research are the low cost of maintenance, little ethical constraints and no biohazard risks. Hence, it has been long recognized as an excellent system for drug screening and safety assessment $[5,6]$. Furthermore, the BmN4 cell line of Bombyx mori [7] has been intensely used in studying many different biological aspects in the laboratory, including virus infection $[8,9]$ and germline piRNA biology [10]. Despite the widespread usage of this ovary-derived cell line, its exact genomic sequence and a tested gene structure model is still missing. Natural sequence variations will interfere with primer design for gene amplification, the design of CRIS PR guides and limit matching of short sequencing reads in transcriptomics as well as peptide coverage in proteomic experiments.

By proteotranscriptomics, combining in-depth transcriptome sequencing and high-resolution mass spectrometry, we establish protein evidence for 6273 genes. Using spatial proteomics, we additionally experimentally classify the localization of several thousand proteins using the recently published LOPIT-DC workflow [11], which utilizes differential ultracentrifugation following removal of unlysed cells to achieve enrichment of cellular compartments in different fractions. With this strategy, not only accurate gene models for the protein-coding genes, but also their subcellular localization is made available. Overall, we here provide a proof of concept for the generation of species-, strain- and cell line-specific gene annotation for protein-coding genes based on experimental evidence without the need of a sequenced genome.

\section{Results}

\section{Transcriptome assembly from RNA-seq data}

We generated 167.8 million paired reads (86.2 million reads from poly-A enriched and 81.6 million from rRNA depleted total RNA samples) by Illumina paired-end sequencing of RNA prepared from the BmN4 cell line of Bombyx mori. To prove our concept of being able to produce a proper annotation without a genome and due to the unclear genetic background of the cell line, we applied a genome-free de-novo approach using the Trinity suite [12] (Fig. 1a). After quality filtering, adapter trimming and erroneous k-mer removal almost $165 \mathrm{M}$ paired

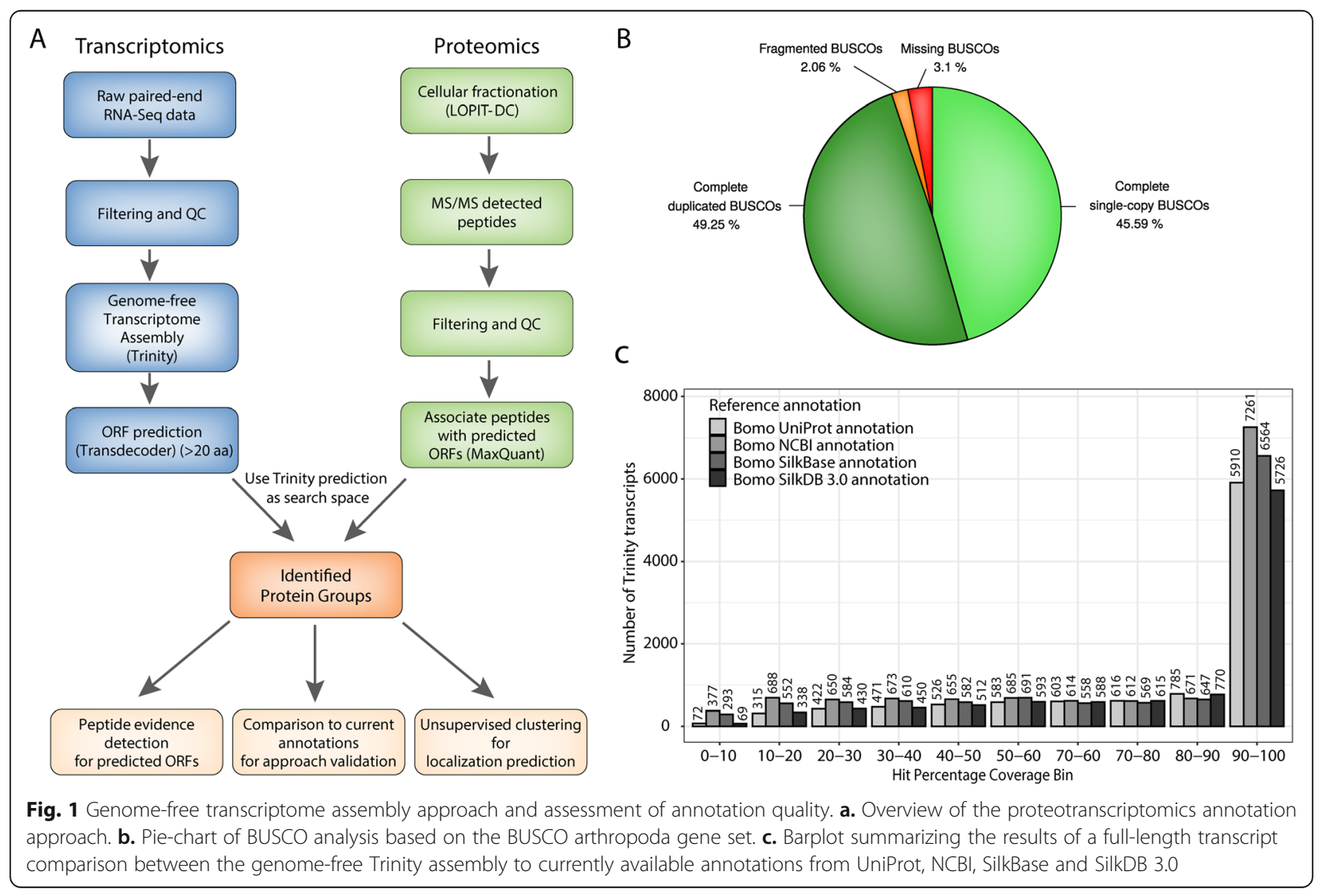


reads and 158,589,380 bases were assembled into 186 , 401 'Trinity transcripts' constituting 120,287 distinct 'Trinity genes'. The assembled raw transcriptome represents $98.32 \%$ of the input reads, which shows that the assembly is highly representative (Additional file 1: Table S1). The traditional N50 statistics describe the minimal transcript length of transcripts that are assembled from at least $50 \%$ of the reads. We found the N50 length for our assembly to be 1553 bases. A better representation excludes lowly expressed transcripts as they might exhibit bigger biases. Hence, we investigated the N50 values across different expression level bins (ExN50) (Additional file 1: Fig. S1). We found that the ExN50 peaks between the 80 and 90 expression percentiles. Thus a better representation is the E90N50 statistic, which represents the minimal transcript length of transcripts in the 90th expression percentile that are assembled from at least $50 \%$ of the reads mapping to these transcripts. The E90N50 transcript contig length is 2270 bases (Additional file 1: Table S2). We used TransRate [13] to validate the quality of the raw assembly (Additional file 1: Table S3). TransRate assesses the accuracy and completeness of a transcriptome assembly using only the input reads. It proceeds by mapping the reads to the assembled contigs, analyzing the alignments, calculating metrics for each individual contig, integrating these contig-level metrics to provide a contig score, and then combining the accuracy of the assembly with the score of each contig to produce an overall assembly score. The crude overall and optimal Transrate assembly score is 0.31 and 0.41 , respectively, of which both are in the 70th percentile range of Transrate assembly scores of 155 published de-novo assembled transcriptomes [13] (Additional file 1: Fig. S2). The expression-levelweighted assembly score, which weights each constituent contig score by the relative abundance level of each contig raises to 0.54 validating the high quality of the assembly and indicating that most of the low TransRate scores stem from contigs that are of relatively low abundance.

To extract all potential protein-coding transcripts from the assembled contigs, we applied TransDecoder [14] and kept transcripts that comprise an open reading frame of at least 20 amino acids. This filtering resulted in a list of 317,031 potential protein-coding open reading frames based on 95,817 individual genes. These potential protein-coding sequences are the basis of our further analysis. Using BUSCO [15], we detected that our assembled protein coding transcripts cover $94.8 \%$ of the arthropod BUSCO gene set (Fig. 1b and Additional file 1: Table S4). Currently, three main initiatives have provided Bombyx mori genome assembly and annotation. For precision estimation, we compared our data to the currently available annotations of the different Bombyx mori varieties from UniProt UP000005204 with 14,776 gene models [16], NCBI Annotation Release 101 with 14,998 gene models, SilkBase 2017 with 16,880 gene models [4] and SilkDB 3.0 with 16,069 gene models [17]. In general, correspondence between current annotations and de-novo predicted proteins is high, with the majority of transcripts sharing protein full sequence coverage (90-100\%) to the respective UniProt, NCBI, SilkBase and SilkDB 3.0 proteins (Fig. 1c). When analyzing the genetic sequence variation between the $\mathrm{BmN} 4$ cell line and the transcripts from the NCBI and SilkBase annotation by mapping the RNA-seq data to the respective CDS sequences of predicted gene models, we found on average an exchange of 1 in 129 bases for NCBI annotations (i.e. 126,300 changes in 16,393,027 bases) and 1 in 105 bases for SilkBase annotations (i.e. 187,534 changes in 19,826,985 bases). The results of both comparisons are highly consistent. Approximately $75 \%$ of the detected changes are silent (synonymous) mutations while around $25 \%$ have missense (non-synonymous) and $0.4 \%$ nonsense effects (Additional file 1: Table S5). These results unveil an unexpected quite large variation between Bombyx mori strains and emphasize the importance of applying a genome-free approach to provide exact CDS sequences especially for molecular biology applications.

Functional annotation of TransDecoder predicted protein sequences was performed using Trinotate [18] including blastp searches against all model species Swissprot databases, HMMER to identify protein domains, signalP to predict signal peptides, tmHMM to predict transmembrane regions and RNAMMER to identify rRNA transcripts. Furthermore, we used deeploc to predict protein localizations from the respective protein sequences. All functional annotations are included in the annotation file (Additional file 2: Table S10).

\section{High resolution mass spectrometry data provides peptide evidence for protein coding transcripts}

In order to provide evidence for the protein coding capability of our predicted protein coding open reading frames (ORF), we performed mass spectrometry measurements of protein extracts from the $\mathrm{BmN} 4$ cell line. Using LOPIT-DC [11] as a strategy to fractionate our sample, we aimed to increase protein detection depth, while also gaining cellular localization information for the detected protein sequences. Our full Bombyx mori proteome data set with 10 fractions contained 3,685,257 MS/MS spectra. Using the predicted open reading frames of the Trinity transcriptome assembly as search space for the mass spectrometry data, we noted a higher rate of identified MS/MS spectra compared to using the four currently available protein annotations from UniProt, NCBI, SilkBase and SilkDB 3.0 (two-sided paired Wilcoxon signed rank test, $p=3.7^{*} 10^{-8}, p=4^{*} 10^{-8}, \mathrm{p}=$ $3.7^{*} 10^{-8}$ and $p=3.71^{*} 10^{-8}$, respectively (Fig. 2a). 


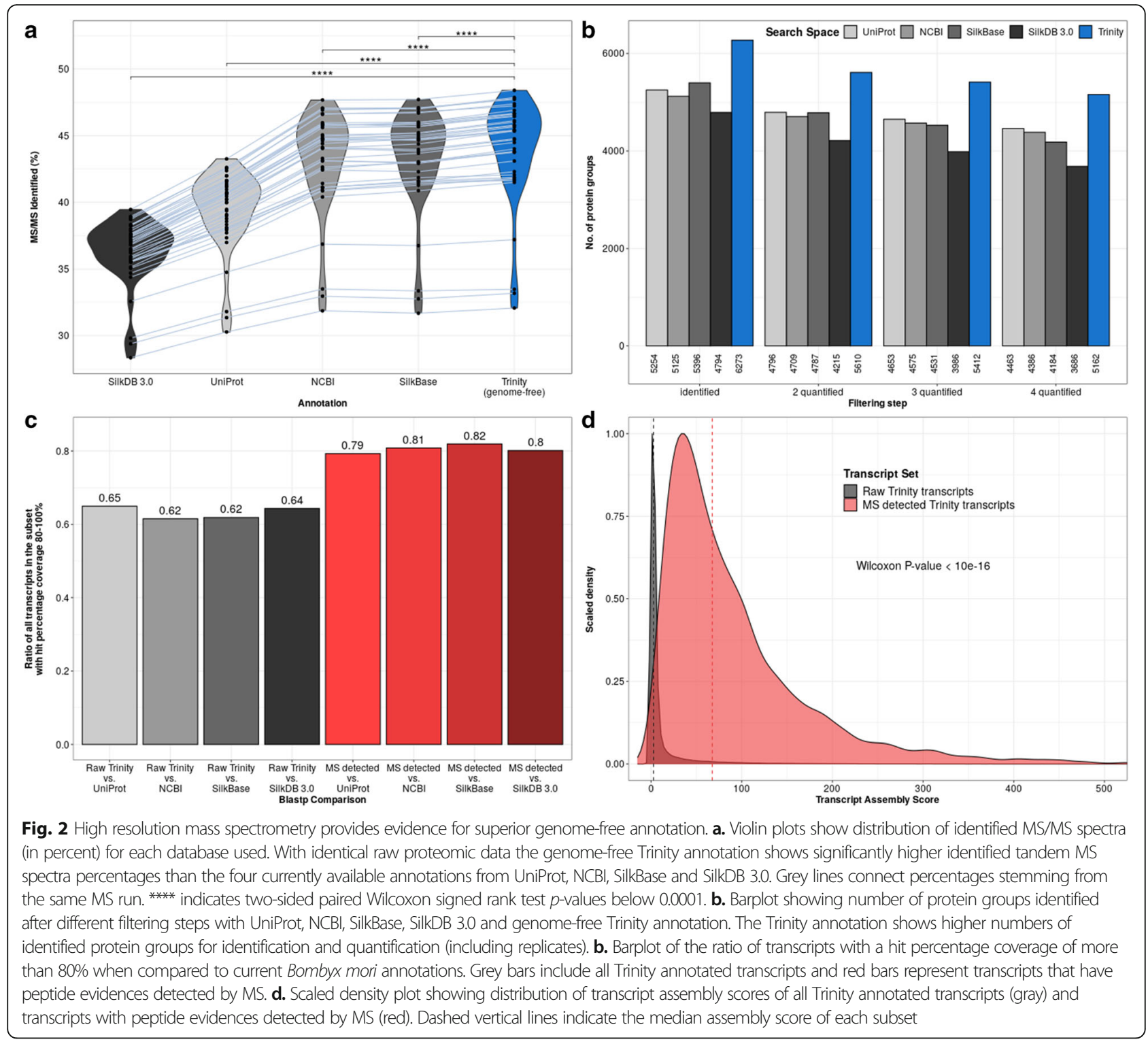

Applying stringent filtering criteria to have at least 2 identified peptides (at least one of them being unique), we identified a total of 6273 protein groups (fasta files of the CDS and protein sequences of these proteins are provided Material S1 and S2, respectively). This was 16, 18,14 and $24 \%$ higher than for the currently available UniProt (5254 identified protein groups), NCBI (5125 identified protein groups), SilkBase (5396 identified protein groups) and SilkDB 3.0 annotations (4794 identified protein groups) (Fig. 2b), emphasizing the power of strain specific gene sequences to increase proteome coverage and also validating the high quality of our genome-free de-novo transcriptome assembly. In order to investigate whether peptide identification could be hindered by provided protein annotations that include strain specific differences in protein sequences, we extracted peptide identification for relevant proteins from both Trinity and the most similar SilkBase annotations and chose as representatives those pairs that have an overlap of more than $80 \%$ in sequence but are not $100 \%$ identical in their protein sequence. We extracted all missense mutations that were identified for these annotation pairs and calculated for the respective locations the proportion of peptides that were not identified in the SilkBase search, although they could be identified in our de-novo Trinity annotation. We found that $88 \%$ of peptides assigned to predicted missense mutations (2325 out of 2653 peptides in 1988 protein groups affected) indeed hamper peptide identification when using the SilkBase annotation as search base.

Detected protein groups show improved quality statistics when compared to the raw in silico predicted 
potential protein coding transcripts, e.g. better overall correspondence with current UniProt, NCBI, SilkBase and SilkDB 3.0 annotations (Fig. 2c, Additional file 1: Fig. S3A), higher assembly scores (Fig. 2d) and longer transcript lengths (Additional file 1: Fig. S3B). We further observed that assembled contigs with high TransRate scores are indeed enriched with identification by mass spectrometry emphasizing the validity of the scoring approach used by TransRate, which is based on the raw read alignment features only (Additional file 1: Fig. S3C).

Although the correspondence between our annotation and the annotation provided by NCBI and SilkBase is overall high (Fig. 2c), there are still almost $20 \%$ of predicted coding sequences that correspond with less than $80 \%$ hit percentage coverage to the current annotations. We noted that some of the current SilkBase annotated transcripts are split into several (mostly two) separated genes in our annotation. This observation can have two main explanations: either the current gene annotations are interdependent (SilkBase includes NCBI annotations in the prediction process) and thus an erroneous annotation from earlier predictions could have been transferred to the newest SilkBase annotation, or, the separation of the non-corresponding annotations in our genome-free approach is wrong. To decide between these two possibilities, we checked the RNA-seq read coverage across the gap between two separated proteins in our annotations that were suggested by SilkBase to be a single protein (941 pairs in total corresponding to 631 SilkBase genes). For this we investigated the RNA-seq reads coverage for each of the relevant pairs of our Trinity predicted proteins and the respective genomic gap between these using the SilkBase genome assembly. Overall we observe that $76 \%$ of the Trinity annotated splits (629 out of 826 protein pairs) are well supported by clear gaps in the RNA-seq raw data alignment at the split site (Fig. S4 A and B). Only 5.5\% of all Trinity predicted proteins (348 unique proteins in 195 protein pairs out of 6273 detected proteins) do not show an evident gap in read coverage and hence likely have been falsely split in the annotation process (Additional file 1: Table S6). Our set of identified ORFs also includes 188 predictions that have a less than $85 \%$ hit coverage with a SilkBase annotation entry. The length differences are shown in Additional file 1: Fig. S5A. In order to investigate if the shorter ORFs are supported by read mapping data, we calculated the difference between the read mapping frequency in the ORF region with the coverage at the edges of the transcripts. 124 (66\% of the short ORFs) identified proteins show an evident absence of reads at the edges of the transcripts, while the remaining 64 might have been falsely split as we could observe mapped reads adjacent to the edges (Additional file 1:
Fig. S4C). Based on these observations, we conclude that our method has a precision rate of at least $93.4 \%$ (5861 out of 6273) for assigning individual genes. The respective categorizations into "high correspondence", "evidently split", "probable false split", "evidently shorter than SilkBase" and "probably falsely shorter than SilkBase" have been included in the annotation table for clarity (Additional file 2: Table S10). Furthermore, we found mass spectrometry evidence for 164 predicted proteins that have been missed in any of the current annotations (marked with "newly annotated" in the annotation Additional file 2: Table S10, peptide evidence information for all novel proteins are provided in Additional file 4: Table S12). Another group of genes (513 genes ( $8 \%$ of all ORFs detected by MS); marked with "longer than SilkBase") are longer than annotated in the current SilkBase annotation, however differences are mostly neglectable (Additional file 1: Fig. S5B). We provide a website (http://butterlab.org/bombyxviewer) which incorporates data regarding all ORFs with mass spectrometric evidence including transcript and protein sequences and a genome viewer based on the SilkBase genome showing gene structure and individual RNA-seq read mapping. Peptide evidence information and annotated MS-MS spectra of newly identified proteins are therewith also provided for download.

\section{Subcellular localization of proteins determined by LOPIT-DC} To assign the Bombyx mori proteins to sub-cellular compartments, we performed label-free quantitative spatial proteomics adapting the recently released LOPIT-DC protocol [11]. Using differential centrifugation steps, we generated 10 subcellular fractions in independent quadruplicates. Fractionation replicates correlate very well (average Pearson correlation coefficient $>0.97$ ) (Fig. 3a) and cluster together in the first two PCA dimensions (Fig. 3b). Within the gradient, fraction series [1-9] are similar to each other, while fraction 10 is most different from all others. This is consistent with fraction 10 constituting an acetone precipitation of all proteins that were not separated in the previous fractionation steps. Analyzing significant changes in pairwise comparisons of all fractions, efficient separation can be recapitulated, i.e. an increasing diversity of proteins can be observed throughout subsequent fractionation steps (Fig. 3c). LFQ data and differential expression statistics across fractionation samples can be found in Additional file 3: Table S11.

We applied an unsupervised clustering approach to detect unique fractionation profiles. Self-organizing map (SOM) clustering of the normalized protein intensities of all proteins showing significant changes in at least one of the pairwise fractionation comparisons and appropriate clustering assessment and filtering (see Methods and Additional file 1: Fig. S6) revealed 8 main 


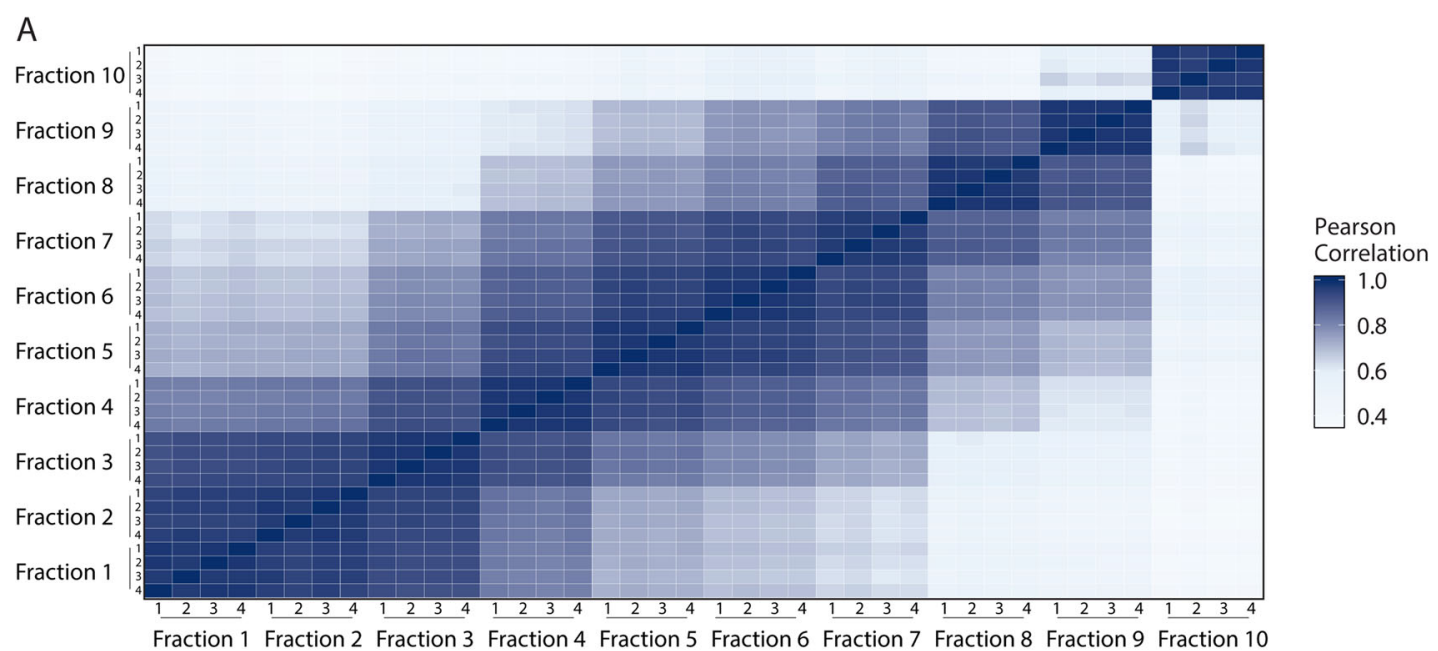

B

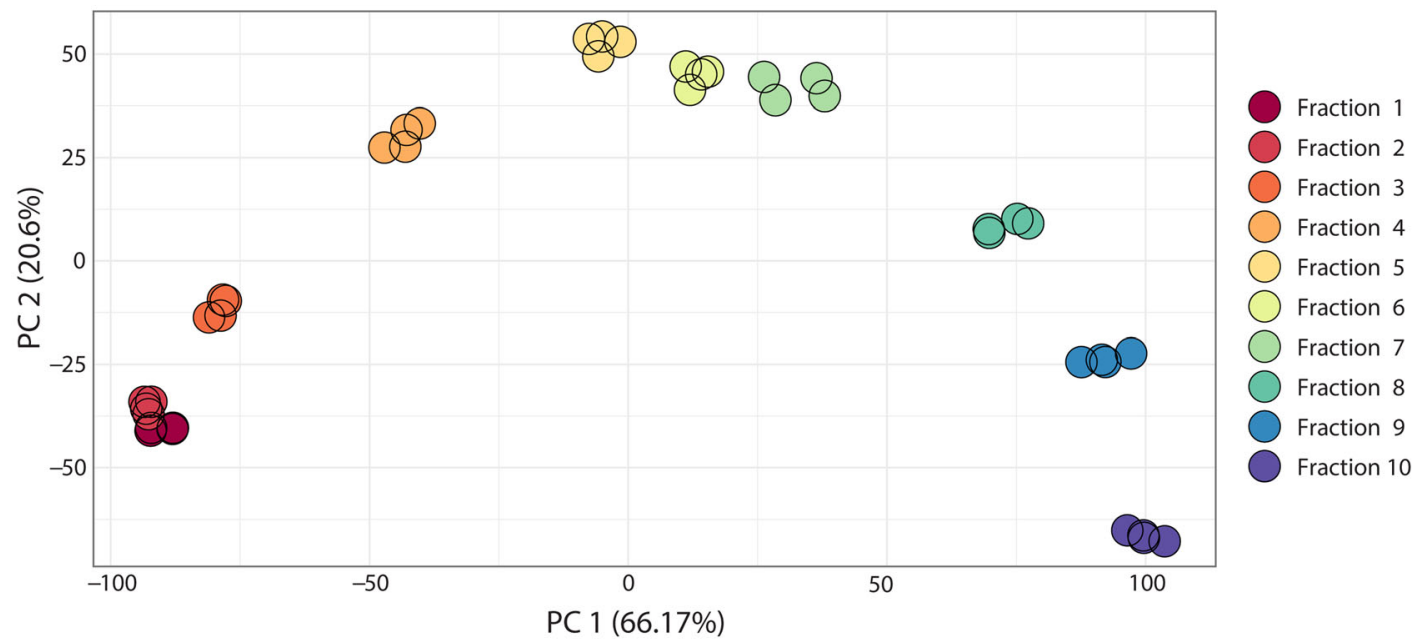

C

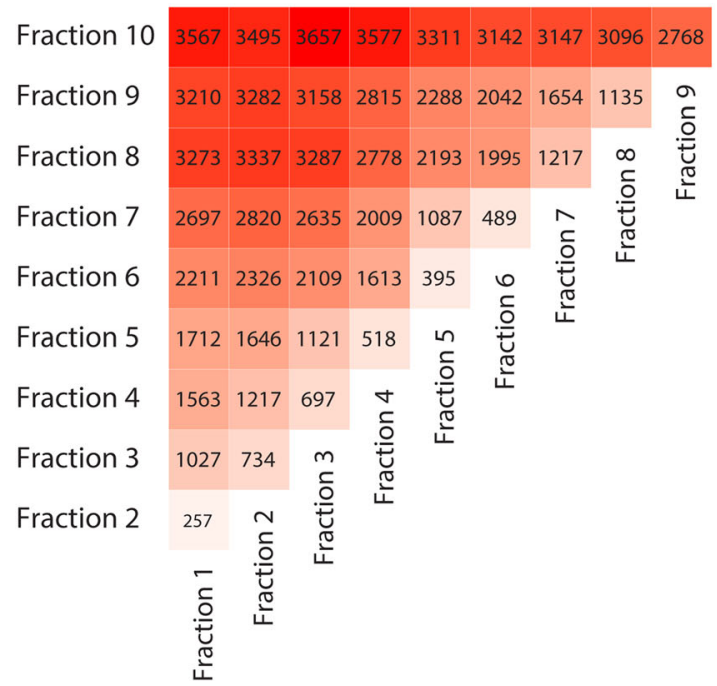

No. proteins with changed protein intensities

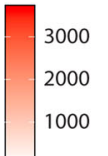

Fig. 3 (See legend on next page.) 
(See figure on previous page.)

Fig. 3 Spatial proteomics unveils subcellular localization of Bombyx mori proteins. a. Heatmap of all pairwise comparisons (pearsons correlations) shows high concordance between replicates and relatedness of adjacent fractions. b. Principal component plot based on the 1000 most dynamic protein groups demonstrates differences of fractions and similarity of replicates. Replicates (same color) cluster together and (except for fraction 1 and 2) farther away from the other fractions. c. Summary heatmap of number of proteins with significantly different protein intensities

across fractions

clusters encompassing 3942 protein groups as depicted in Fig. 4a. The mean expression profiles across fractionation of the different clusters are depicted in Fig. 4b (and in Additional file 1: Fig. S7 for individual genes). In order to characterize the different clusters in terms of their potential subcellular localization or function, enrichment of specific categories as determined by signalP (prediction of signal peptides), tmHMM (prediction of transmembrane regions) and the Gene Ontology cellular-component (GO_cc) annotation were calculated (Fig. 4c, Additional file 1: Table S7). Clusters 1-4 represent proteins that show relatively high intensities in the early fractionation steps with diminished intensities in later steps. Generally, membrane associated proteins (framed with black box in Fig. 4c) are highly enriched in clusters 2 and 3 . To get a more specific insight into the subcellular localization, we subsequently checked for enrichment of the GO_cc terms 'lysosome', 'peroxisome', 'golgi apparatus', 'nucleus', 'chromatin', 'endoplasmic reticulum' (ER), 'mitochondrion' and 'ribosomes', which were inferred by orthology to well-annotated model organisms in each cluster (framed with red box in Fig. 4c). The most prominent enrichment was observed for cluster 1, which exhibits high levels in the first three fractions and low levels in later fractions. This cluster is highly enriched with mitochondrial genes (Fisher's exact test, $P=10^{-197}$, fold-enrichment $=35.56$ ). Cluster 2 shows reduced intensity after fractionation step 4 and is enriched with peroxisome, ER and lysosome annotated proteins (Fisher's exact test, $P=10^{-16}, P=10^{-6}$ and $10^{-5}$, fold-enrichment $=24.14,3.2$ and 4 , respectively). Cluster 3 has lower intensity starting at fractionation step 5 and represents mainly endosplasmatic reticulum (ER) proteins (Fisher's exact test, $P=10^{-7}$, foldenrichment $=3.37$ ). The profile of cluster 4 shows reduction of protein intensities after fractionation step 8 and contains proteins from Golgi apparatus (Fisher's exact test, $P=10^{-5}$, fold-enrichment $=2.9$ ). The second highest enrichment could be observed for cluster 5, where measured protein intensities peak in fraction 7-9 and which is highly enriched with ribosomal 40 s and 60 s proteins (Fisher's exact test, $P=10^{-25}$, foldenrichment $=11.76$ ). Cluster 6 encompasses proteins with low abundance in the initial fractionation steps, which increase until step 9 and decline to minimal levels in fraction 10. This cluster exhibits a mixed enrichment profile of nuclear and chromatin associated proteins, but also with ribosomal proteins (Fisher's exact test, $P=$

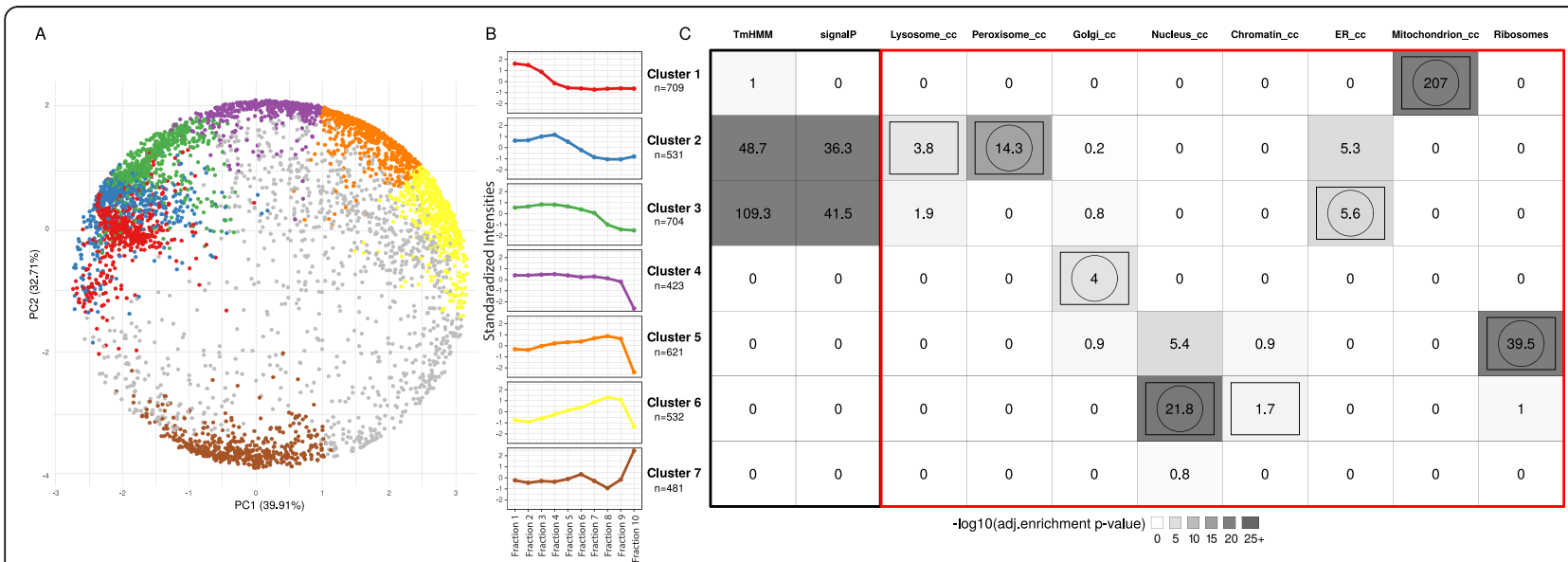

Fig. 4 Unsupervised clustering of fractionation mass spectrometry data. a. Principal component plot of components 1 and 2 calculated from standardized average protein intensities. Standardized protein intensities across fractionation samples were clustered using SOM (self-organizing maps). Proteins in PCA space are colored according to their assignment to one of eight distinct clusters (see color code in b). 795 proteins could not be associated with any of the clusters (gray colored dots). b. Line plots of the median standardized protein intensities across fractionation steps of the different clusters assigned by SOM (see a). c. Heatmap summarizing enrichment analyses of cellular localization annotations in the respective clusters. Color darkness corresponds to levels of enrichment (- $\log 10$ of adjusted $P$-values). Rectangles and circles indicate highest enrichment for the corresponding localization and gene clusters, respectively. General and more specific localization categories are framed in black and red, respectively 
$10^{-14}, P=10^{-3}$ and $P=10^{-13}$, fold-enrichment $=2.62$, 2.97 and 6.92, respectively). Cluster 7 is the only cluster, that show exclusively high enrichment with nucleus associated proteins (Fisher's exact test, $P=10^{-19}$, foldenrichment $=3.18$ ). Cluster 8 , which shows abrupt protein intensity increase in the last two fractions couldn't be associated with any of the known localizations and hence was not further analyzed. Overall the cellular localization profiles of the different clusters correspond very well (average Spearman's correlation coefficient $=$ 0.88 ) with those established in the LOPIT-DC method paper [11] where the assignment was established using experimentally validated marker genes (Additional file 1 : Fig. S8). Further, orthologous proteins of established Drosophila melanogaster cellular compartment marker genes show highly coherent profiles to the ones established by our unsupervised approach (Additional file 1: Fig. S9). This shows that the fractionation strategy is robust and widely applicable. Comparing the fractionation profile of each protein to each of the clusters enables localization prediction also for proteins that could not be annotated properly by previous in silico analysis. The resulting clusters allow to assign proteins especially to the following 6 compartments (in descending order of certainty): Mitochondria (cluster 1), Ribosome (cluster 5), Nucleus (cluster 7), Peroxisome (cluster 2), Endoplasmic reticulum (cluster 3) and Golgi apparatus (cluster 4). For each individual MS detected protein, we calculated the correlations between its expression profile across fractionations and the predicted median profile of the above depicted localization. These correlation values are provided as confidence score for the localization probability of each protein. The information for each detected protein, including all relevant information such as transcripts type, length, score, annotations and localization categorization weights are provided in Additional file 2: Table S10). All CDS and protein sequences of the assembled and identified proteins are provided in Additional files 5 and 6 .

\section{Discussion}

We here show that by combining comprehensive RNAseq data and high-resolution mass spectrometry data, we achieve a comparable and even slightly better annotation of protein-coding genes in Bombyx mori than previous efforts based on genome or transcriptome guided denovo strategies. Even in the comparison of our genomefree to our own genome-guided assembly using the same raw RNA-seq and mass spectrometry data a slightly better performance can be observed in the genome-free approach (see Additional file 1: Table S9). This fact emphasizes the importance of using genome-free approaches in conditions were provided genomes are suspected to stem from different genetic backgrounds as the measured system.

Our extensive comparison between our genome-free proteotranscriptomics annotation and the provided annotations from UniProt, NCBI, SilkBase and SilkDB 3.0 showed that UniProt and SilkDB 3.0 currently provides the annotation with the weakest performance in $\mathrm{BmN} 4$ cells. Gene annotations provided by NCBI and SilkBase are more comprehensive, however still do not report the full protein-coding potential demonstrated by the elevated percentage of identified MS-MS spectra in our tailor-made assembly (Fig. 2a) and the identification of 164 new proteins in this study. As BmN4 cells were derived from the ovary of a female animal, we were curious to know if any of the newly identified proteins might be located on the female-determining Bombyx mori chromosome W [19]. Mapping the CDS sequences of the respective proteins to the sequences of the $\mathrm{W}$ chromosome did not result in any localization, while they did map rather evenly dispersed to all other chromosomes (see Additional file 1: Table S10). In fact, none of the identified proteins mapped to chromosome $\mathrm{W}$ sequences recapitulating the current knowledge that this chromosome is depleted of protein coding genes [20]. Although the correspondence between our annotation and NCBI and SilkBase is overall high, there are still $20 \%$ of coding sequences with less than $80 \%$ hit length, mostly attributed to split genes in our assembly. Performing a detailed investigation of the gap region, we could provide evidence that in $80 \%$ of cases the split version in our assembly is supported, improving gene models for 451 genes.

Many studies have shown that small proteins $(\leq 100$ amino acid residues) can be involved in important biological processes, including cell signaling, metabolism, and growth [21]. However, they are underrepresented in many genome annotations as they are notoriously hard to predict because of their small ORF size [21]. To validate these small peptides, we kept all ORF with at least 20 amino acids. Indeed, we detected 308 small proteins (5\% of all protein groups identified) with at least two peptides (one of them unique) and many of them reaching relatively high expression levels, however the overall expression levels are lower than for longer proteins (Additional file 1: Fig. S10).

Additionally, we used the recently published LOPITDC approach [11] to provide experimental data for localization of our detected proteins. The high correspondence to the LOPIT-DC results, despite changing from TMT to LFQ and using cell lines from different species (human osteosarcoma U2OS vs. B. mori BmN4), indicate a universal applicability of the fractionation protocol and the resulting data. The resulting clusters allow assigning proteins especially to the following 
compartments: Mitochondria, Ribosome, Nucleus, Peroxisome, Endoplasmic reticulum and Golgi apparatus.

The current fractionation approach allowed us to detect peptide evidence for more than 6200 proteins. If more comprehensive databases are of interest, these limits may be overcome by using more diverse conditions or several different tissues for extraction of transcriptomic and proteomic data. While we here provide annotation for Bombyx mori, this approach is readily applicable to any species, including more complex organisms such as vertebrates and plants. While possible parameters might need to be adapted such as even deeper RNA-seq data, the high fraction of non-coding genome regions or highly repetitive structure that make genome assembly challenging can be disregarded.

Our developed proteotranscriptomics approach improves current gene annotations and provides the exact gene sequences for other applications such as gene amplifications via cDNA or planning CRISPR guides around the translation start site.

Importantly, the proposed annotation approach readily works without any genome reference and hence provides a precise, time- and cost-efficient method to construct annotations for protein-coding genes in any species where properly sequenced genomes are still out of reach.

\section{Conclusions}

Combining in-depth transcriptome sequencing and high resolution mass spectrometry, we here use proteotranscriptomics to improve gene annotation of proteincoding genes in the Bombyx mori cell line BmN4 which is an increasingly used tool for the analysis of piRNA biogenesis and function. Using this approach we provide the exact coding sequence and evidence for more than 6200 genes on the protein level. We show that proteotranscriptomics is an efficient, cost-effective and accurate approach to improve previous annotations or generate new gene models. As this technique is based on de-novo transcriptome assembly, it provides the possibility to study any species also in the absence of genome sequence information.

\section{Methods}

\section{Experimental design}

To build a genome-free proteotranscriptomics-based gene annotation we combined two types of data. First, RNA-seq data of polyadenylated mRNA entities combining poly-A enriched and rRNA depleted samples from Bombyx mori BmN4 cell line was used to predict potential protein-coding genes. Secondly, MS/MS spectra data was used to find evidence for the predicted proteincoding genes. Profiling subcellular localization of proteins in Bombyx mori cells was performed using the LOPIT-DC strategy [11]. The procedures were performed with four biological replicates based on the high level of reproducibility between replicates (average Pearson correlation coefficient $>0.97$ ). Results are represented as averages of the biological replicates. We used Trinity for genome-free de novo RNA assembly and the MaxQuant data analysis platform [22] for quantitative proteomics analysis.

\section{Cell propagation and RNA extraction}

The Bombyx mori larval ovary-derived cell line BmN4 (RRID:CVCL_Z634) [7] was kindly provided by the Ketting group (Institute of Molecular Biology, Mainz, Germany)). Cells were cultured in Insect media IPL-40 (Pan Biotech) with 10\% heat-inactivated FBS (Sigma) and $1 \mathrm{x}$ Penn-Strep (Sigma) at $27^{\circ} \mathrm{C}$.

For RNA-sequencing total RNA was extracted from 10 million cells using the RNAeasy Mini Kit (Qiagen) according to standard protocol. RNA integrity was tested by agarose gel electrophoresis and Bioanalyzer (RNA Nano Assay). RNA was quantified using Qubit.

\section{RNA-seq measurements}

RNA-sequencing libraries were prepared from total RNA using two different RNA enrichment protocols: 1. poly(A) purification using Illumina TruSeq stranded mRNA LT Sample Prep Kit following Illumina's standard protocol (Part \# 15031047 Rev., E). [polyA-enriched] 2. depletion of ribosomal RNA using Illumina TruSeq stranded Total RNA LT Sample Prep Kit following Illumina's standard protocol (Part \# 15031048 Rev. E) [ribo-minus].

The libraries were prepared with a starting amount of $1000 \mathrm{ng}$ and amplified in 10 PCR cycles and profiled using a DNA 1000 Chip on a 2100 Bioanalyzer (Agilent technologies) and quantified using the Qubit dsDNA HS Assay Kit, in a Qubit 2.0 Fluorometer (Life technologies). The two libraries were pooled together in equimolar ratio and sequenced on 1 NextSeq 500 Midoutput FC, PE for $2 \times 79$ cycles plus 7 cycles for the index read. The measurements of polyA-enriched and ribo-minus RNA resulted in $86,178,436$ and $81,597,503$ paired-end reads of length $80 \mathrm{bp}$, respectively. We assayed mycoplasma contamination by aligning all raw RNASeq forward reads to the genomes of A.laidlawii, M.arginini, M.fermentans, M.hominis, M.hyorinis and M.orale. The maximum percentage of uniquely mapped reads is below $0.00026 \%$ and therefore a contamination can be excluded (see Table S8).

\section{Transcriptome assembly}

The two RNA-seq datasets (polyA-enriched and ribominus RNA) were used in combination to assemble the transcriptome. First, both raw fastq files were cleaned from erroneous k-mers using Rcorrector [23] and the specialized scripts from TranscriptomeAssemblyTools 
(FilterUncorrectablePEfastq.py). Secondly, adapter sequences were removed using TrimGalore (a wrapper around Cutadapt [24] and FastQC [25]) and reads were filtered to include only pairs consisting of proper pairs of minimum length of 36 nts each. These clean-up steps removed only $2 \%$ of the paired reads. The fastq files were then combined. For the genome-guided assembly raw RNA-seq data was mapped to the Bombyx mori genome provided by SilkBase (http://silkbase.ab.a.u-tokyo. ac.jp/cgi-bin/index.cgi) using STAR (version 2.5.4b) [26]. The raw RNA-seq or mapped data was used for a genome-free de novo or genome-guided assembly approach using the Trinity suite (Trinity version 2.4.0) [12] with the following parameter setting: [for genome-free: --seqType fq --SS_lib_type RF --min_kmer_cov 1], [for genome-guided: Trinity -genome_guided_bam -genome_guided_max_intron 30,000 --genome_guided_min coverage 2]. The resulting Trinity fasta files were then further processed with TransDecoder version 5.4.0 [14] to predict potential protein coding transcripts using a length threshold of 20 amino acids. The resulting peptide fasta files were used as search space in subsequent steps for mass spectrometry data analysis.

\section{Quality check of transcriptome assembly}

The quality of the assembled transcriptome was assessed using several different state of the art approaches. These included general metrics of number of assembled transcripts, mean, median and Ex90N50 transcript lengths. The alignment rate of the raw reads to the assembly was calculated using Bowtie2 (version 2.3.4.3) [27] and dedicated scripts provided by Trinity (Trinity version 2.4.0) [12]. BUSCO (version 2.0) [15] with the arthropodae BUSCO database was used to assess the completeness of the assembly. Transrate scores and additional quality metrics were established using TransRate (version 1.0.3) [13]. Coherence with current annotations was measured using a combination of blastp (BLAST + version 2.8.1) [28] and Trinity tools (Trinity version 2.4.0) [12]. For RNA-seq coverage validations the combined cleaned RNA-seq data was mapped to the SilkBase genome assembly using STAR (version 2.5.4b) [26]. Coverage per base was calculated using bedtools (version 2.26.0) [29] using the -pc option to also account for intronic alignment. Then using customized $\mathrm{R}$ (version 3.5.3) [30] scripts the average coverage per transcript or gap region was extracted (Fig. S4).

\section{Annotation of identified transcripts}

Functional and domain annotations were produced using Trinotate (version 3.1.1) [18] combining the following applications: HMMER (version 3.2.1) [31] to identify protein domains, signalP (version 5.0) [32] to predict signal peptides, TMHMM (version 2.0c) [33] to predict transmembrane regions, RNAMMER (version1.2) [34] to identify rRNA transcripts in addition to infer Gene Ontology and KEGG terms from orthologies established by BLAST+ (version 2.8.1) [28] with a swissprot database of all major model species. Further, localization predictions from protein sequences of the assembly were calculated using deeploc (version 1.0) [35].

\section{Detection of variation level between NCBI and SilkBase protein coding sequences and BmN4 RNASeq data}

In order to characterize the level of variation between the Bombyx mori coding sequences in the NCBI annotation and the BmN4 specific transcriptome, we first mapped the RNA-seq data also used for transcriptome assembly to NCBI coding sequences using STAR aligner (version 2.5.4b) [26]. We then used GenomeAnalysisTK (version 3.8-0-ge9d806836) [36] to extract sequence variation information into a vcf file. SnpEff (version 4.4) [37] was used to annotate all sequence changes regarding their type (single nucleotide polymorphism (snp), deletion, insertion), their impact (low, moderate, high severeness) and their functional class (missense, nonsense, silent mutation). Results of this analysis are summarized in Table S5.

\section{Subcellular fractionation}

Subcelluar fractionation of Bombyx mori cells was performed in quadruplicates and based on the LOPIT-DC strategy [11] with some modifications. Per replicate 70 million BmN4 cells were collected and suspended in 4 $\mathrm{ml}$ lysis buffer (0.25 M sucrose, $10 \mathrm{mM}$ HEPES pH 7.5, 2 mM EDTA, 2 mM Magnesium acetate, Roche complete protease inhibitors). Cells were dounced in a $7 \mathrm{ml}$ glass douncer with 50 strokes using a type B pestle. Samples were fractionated using centrifugation speed and times as indicated in the table below following the LOPIT-DC strategy [11]. For centrifugation steps a Heraeus Fresco 21 centrifuge (Thermo) or a Optima Max-XP benchtop centrifuge (Beckmann) with a TLA 100.3 rotor were used. Supernatant of the 9th fractionation step was precipitated using ice cold acetone and the pellet was resuspended in $50 \mathrm{mM}$ HEPES/KOH pH 7.9 (represents fraction 10).

\section{Mass spectrometric sample preparation and measurement}

$50 \mu \mathrm{g}$ protein from each fraction were loaded on a $4-$ 12\% NOVEX Bis-Tris PAGE gel (Thermo) and separated for $7 \mathrm{~min}$ at $180 \mathrm{~V}$ in $1 \mathrm{x}$ MES buffer (Thermo). Proteins were fixated and stained with Coomassie. After destaining with water, in-gel digest preparation and MS stage tip purification were performed as previously described [38], [39]. Peptides were analyzed by nanoflow liquid chromatography on an EASY-nLC 1000 system 
(Thermo) coupled to a Q Exactive Plus mass spectrometer (Thermo). Peptides were separated on a C18reversed-phase column packed with Reprosil aq1.9 (Dr. Maisch $\mathrm{GmbH}$ ), directly mounted on the electrospray ion source of the mass spectrometer. We used a ca. 200 min gradient from 2 to $60 \%$ acetonitrile in $0.1 \%$ formic acid at a flow of $225 \mathrm{~nL} / \mathrm{min}$. The Q Exactive Plus was operated with a Top10 MS/MS spectra acquisition method per MS full scan.

\section{Protein identification and label free quantification of protein intensities}

MaxQuant (version 1.5.2.8) [22] was used for raw file peak extraction and protein identification against the following databases individually: UniProt UP000005204 (14,776 entries) [16], NCBI Bombyx mori Annotation Release 101 (14,998 entries), SilkBase 2017 (16,880 entries) [4], SilkDb 3.0 (16,069 entries) [17] or our Trinity-based ORF library (317,031 entries). Protein quantification was performed with MaxQuant using the label free quantification (LFQ) algorithm [40]. The following parameters were applied: trypsin as cleaving enzyme; minimum peptide length of seven amino acids; maximal two missed cleavages; carbamidomethylation of cysteine as a fixed modification; Nterminal protein acetylation and oxidation of methionine as variable modifications. Peptide mass tolerance was set to $20 \mathrm{ppm}$ and $7 \mathrm{ppm}$ was used as MS/MS tolerance. Further settings were: "label-free quantification" with "FastLFQ" disabled, "match between runs" with time window 0.7 min for matching and 20 min for alignment; peptide and protein false discovery rates (FDR) were set to 0.01; common contaminants (standard MaxQuant contaminant list including trypsin, keratin etc.) were excluded. Detailed settings are available in the respective parameter files (uploaded to ProteomeXchange (www.ProteomExchange.org) via the PRIDE [41] partner repository with the dataset identifier PXD014626). MaxQuant LFQ data was further processed using in-house developed tools based on R (version 3.5.3) [30]. This included filtering out marked contaminants, reverse entries and proteins only identified by site. Protein groups with no unique and less than two peptides were removed. Protein group averages were calculated from proteotypic peptides. Prior to imputation of missing LFQ values with a beta distribution ranging from 0.1 to 0.2 percentile within each sample, the values were $\log 2$ transformed. For protein groups consisting of more than one Trinity annotation, we chose the longest as representative of the group for further analysis.

\section{LFQ data analysis and unsupervised clustering}

For overall statistical quality calculations, we calculated proportions of assembly scores, transcript lengths and correspondences with UniProt, NCBI, SilkBase and SilkDB 3.0 Bombyx mori annotations. For clustering purposes, we focused only on protein groups that have four measured LFQ levels in at least one of the fractions and showed significant enrichment in at least one of the pairwise fraction comparisons ending up in 5058 out of 5610 protein groups (90\%). The mean LFQ data of the four biological replicates data was standardized proteingroup-wise by adding the mean of all average fraction intensities and dividing by the standard deviation between all average fraction intensities of the respective protein group. An unsupervised machine learning approach was used to cluster all standardized profiles. We applied the kohonen $\mathrm{R}$ package [42] to build a selforganizing-map (SOM), i.e. to build an artificial neural network that is trained using unsupervised learning to produce a two-dimensional, approximated grouping of the input profiles. The standardized data was initially assigned to a SOM matrix of 12 basic clusters. After combining four clusters with high intra-cluster differences (mean differences above the 75\%-ile intra-cluster distances of all protein groups; see Additional file 1: Fig. S6) into one cluster with uncategorized profiles, eight distinct clusters representing similar profiles remained. These clusters were ordered according to euclidean distances in the first two-component PCA space using the TSP (travelling salesman problem) $\mathrm{R}$ package (version 1.1-7) [43]. Using localization data retrieved through orthology and sequence screening approaches as described earlier, we checked for enrichment of categories relevant for cellular localization, namely $\mathrm{TmHH}$ (sequence based transmembrane region predictions), signalP (sequence based signal peptides identification), Gene Ontology associations based on blast orthology associations for cellular component annotations lysosome, peroxisome, golgi, nucleus, chromatin, endoplasmic reticulum (ER), mitochondrion and ribosome (based on $40 \mathrm{~S}$ and $60 \mathrm{~S}$ ribosomal proteins) annotations. Enrichment scores of each category and each fractionation profile cluster were calculated using Fisher's Exact test (one-sided test, alternate-hypothesis: cluster genes contain more genes belonging to the tested category than non-cluster genes). Correlation of the identified cluster profiles were compared to the profiles of the same cellular localization categories from the original LOPIT-DC paper [11] by calculating the spearman correlation between the median standardized LFQ profiles of our data and the standardized median TMT profiles of the TMT data from human osteosarcoma U-2 OS cell line across fractionations (normalized TMT data is provided in Supplementary data 1 of previous publication [11]). Respective profiles and correlations are shown in Additional file 1: Fig. S8. Drosophila melanogaster cellular compartment marker genes were downloaded from the pRoloc bioconductor package [44] and blasted against our denovo protein database using blastp (BLAST + version 
2.8.1) [28] to find the respective orthologous genes. Newly identified genes were mapped to SilkDB 3.0 genome assembly and sequences of the W chromosomes using gmap version 2019-03-04 [45]. W chromosome sequences were downloaded from GenBank nucleotide database accession numbers AB251908-AB251914.

\section{Supplementary information}

Supplementary information accompanies this paper at https://doi.org/10. 1186/s12864-020-07088-7.

\section{Additional file 1 Supplemental Fig. S1. Distribution of length} distributions across RNA expression level bins. Supplemental Fig. S2 Comparison of Transrate assembly scores to other publicly available assemblies. Supplemental Fig. S3. Overall MS detected transcripts show improved assembly features. Supplemental Fig. S4. RNA-Seq coverage assay for the detection of falsely split genes. Supplemental Fig. S5. Densityplot of deviations from SilkBase annotation for longer annotated genes. Supplemental Fig. S6. Intracluster distances of SOM clusters can be used to filter out clusters that have high variability within the cluster. Supplemental Fig. S7. Depiction of expression profiles of all genes separated into respective clusters. Supplemental Fig. S8: Comparison of fractionation profiles of the respective cellular compartments in original LOPIT-DC TMT data and our Proteotranscriptomics LC-MS-MS approach. Supplemental Fig. S9. Comparison of fractionation profiles of orthologs of established Drosophila melanogaster cellular compartment markers to the mean dynamics determined by SOM clustering and enrichment analysis. Supplemental Fig. S10. Comparison of LFQ expression levels of detected protein groups that are shorter (small proteins) or longer than 20 amino acids. Supplemental Table S1. Read representation statistics of the Trinity assembly. Supplemental Table S2. Expression bins and transcript lenghts. Supplemental Table S3. TransRate analysis results. Supplemental Table S4. BUSCO analysis results. Supplemental Table S5. Bombyx mori NCBI and SilkBase based BmN4 variome. Supplemental Table S6. Statistics of correspondence and differences between genome-free and SilkBase annotations. Supplemental Table S7. Table including enrichment values for all clusters and all cellular localization categories. Supplemental Table S8. Mycoplasma contamination assay. Supplemental Table S9. Comparison of genome-free and genomeguided assembly. Supplemental Table S10. Mapping statistics of newly identified protein CDS sequences to the sequences of the femaledetermining chromosome $\mathrm{W}$

Additional file 2 Table S10. Functional annotation and characteristics of ORFS

Additional file 3 Table S11. Label-free quantification data.

Additional file 4 Table S12. Novel protein evidence data.

Additional file 5. BOMOPTA annotation CDS sequences.

Additional file 6. BOMOPTA annotation protein sequences.

\section{Abbreviations}

BmN4: Bombyx mori N4 cell line; piRNA: Piwi-interacting RNA; RNA-seq: RNAsequencing; CRISPR: Clustered regularly interspaced short palindromic repeats; LOPIT-DC: Localisation of organelle proteins by isotope tagging after differential ultracentrifugation; rRNA: Ribosomal RNA; BUSCO: Benchmarking Universal Single-Copy Orthologs; CDS: Coding sequences;

CDNA: Complementary DNA; ORF: Open reading frame; MS/MS: Tandem mass spectrometry; PCA: Principal component analysis; SOM: Self-organizing maps; GO: Gene Ontology; GO_cc: Gene Ontology cellular compartment; ER: Endoplasmic reticulum; TMT: Tandem mass tag; LFQ: Label-free quantification; TSP: Traveling salesman problem

\section{Acknowledgements}

We are indebted to Walter Bronkhorst for advice on culturing BmN4 cells that were kindly provided by the Ketting group (Institute of Molecular Biology, Mainz, Germany). The RNA sequencing was conducted at the IMB Genomics Core Facility. Parts of this research were conducted using the supercomputer Mogon and advisory services offered by Johannes Gutenberg University Mainz (hpc.uni-mainz.de), which is a member of the AHRP (Alliance for High Performance Computing in Rhineland Palatinate, www.ahrp.info) and the Gauss Alliance e.V.. We gratefully acknowledge the computing time granted on the supercomputer Mogon at Johannes Gutenberg University Mainz (hpc.uni-mainz.de) and Christian Meesters for helpful instructions regarding the implementation of Trinity and Transdecoder on Mogon. We thank Mario Dejung from the Proteomics Core Facility at the Institute of Molecular Biology for setting up the web-server and the dedicated data viewer on the website http://butterlab.org/ bombyxviewer.

\section{Authors' contributions}

$M L, M S$ and FB conceived the project. MS and FB designed the experiments. $\mathrm{ML}$ analyzed the data. MS conducted the experiments. ML and FB wrote the paper. All authors approved the final manuscript.

\section{Funding}

The study was supported by IMB (Institute of Molecular Biology) core funding. The funder had no role in the design of the study, analysis, interpretation of data and in writing the manuscript.

\section{Availability of data and materials}

RNA-sequencing raw data of polyadenylated mRNA entities has been submitted to the Sequence Read Archive (SRA) under SRA identifier SRR9685281.

The mass spectrometric proteomics data including all raw and processed data files and MS/MS spectra files has been deposited to the

ProteomeXchange Consortium via the PRIDE partner repository [41] with the dataset identifier PXD014626.

Genome sequences of the mycoplasma species A. laidlawil (Acholeplasma_laidlawii_pg_8a.ASM1878v1.dna.toplevel), M. arginini (Mycoplasma_arginini_7264.version_1.0.dna.toplevel), M. fermentans (Mycoplasma_fermentans_pg18.ASM20973v1.dna.toplevel), M. hominis (Mycoplasma_hominis_atcc_23114.ASM8586v1.dna.toplevel) and M. hyorinis (Mycoplasma_hyorhinis_sk76.ASM31363v1.dna.toplevel) were downloaded from Ensemble ftp site (ftp.ensemblgenomes.org). The genome sequence of M. orale (GCF_000420105.1_ASM42010v1_genomic) was downloaded from the NCBI ftp site (ftp.ncbi.nlm.nih.gov).

The UniProt proteome of Bombyx mori was downloaded from UniProt database with accession number UP000005204.

The NCBI genome of Bombyx mori was downloaded from NCBI database using assembly ID ASM15162v1, the NCBI proteome was downloaded using Annotation Release ID 101.

The SilkBase genome assembly (Nov.2016) and protein models (Jan.2017) was downloaded from http://silkbase.ab.a.u-tokyo.ac.jp/cgi-bin/download.cgi. The SilkDB 3.0 genome assembly (chromosome.fa.tar.gz) and protein sequences (protein.fa.tar.gz) were downloaded from https://silkdb. bioinfotoolkits.net/base/download/-1.

W chromosome sequences were downloaded from GenBank nucleotide database using accession numbers AB251908-AB251914.

All CDS and protein sequences of the assembled and identified proteins are provided in Additional files 5 and 6 . The complete Trinity-based ORF database can be downloaded from http://butterlab.org/bombyxviewer.

Ethics approval and consent to participate Not applicable.

\section{Consent for publication}

Not applicable.

\section{Competing interests}

The authors declare that they have no competing interests.

Received: 14 April 2020 Accepted: 21 September 2020

Published online: 06 October 2020

\section{References}

1. Mita K, Kasahara M, Sasaki S, Nagayasu Y, Yamada T, Kanamori H, et al. The genome sequence of silkworm Bombyx mori. DNA Res. 2004;11:27-35. https://doi.org/10.1093/dnares/11.1.27. 
2. Xia Q, Zhou Z, Lu C, Cheng D, Dai F, Li B, et al. A draft sequence for the genome of the domesticated silkworm (Bombyx mori). Science. 2004;306: 1937-40. https://doi.org/10.1126/science.1102210..

3. The International Silkworm Genome Consortium. The genome of a lepidopteran model insect, the silkworm Bombyx mori. Insect Biochem Mol Biol. 2008:38:1036-45. https://doi.org/10.1016/J.IBMB.2008.11.004.

4. Kawamoto M, Jouraku A, Toyoda A, Yokoi K, Minakuchi Y, Katsuma S, et al. High-quality genome assembly of the silkworm Bombyx mori. Insect Biochem Mol Biol. 2019;107:53-62. https://doi.org/10.1016/J.IBMB.2019.02. 002.

5. Nwibo DD, Hamamoto H, Matsumoto Y, Kaito C, Sekimizu K. Current use of silkworm larvae (Bombyx mori) as an animal model in pharmaco-medical research. Drug Discov Ther. 2015;9:133-5. https://doi.org/10.5582/ddt.2015. 01026.

6. Abdelli N, Peng L, Keping C. Silkworm, Bombyx mori, as an alternative model organism in toxicological research. Environ Sci Pollut Res. 2018;25: 35048-54. https://doi.org/10.1007/s11356-018-3442-8.

7. Grace TD. Establishment of a line of cells from the silkworm Bombyx mori. Nature. 1967;216:613

8. Katsuma S, Kawamoto M, Shoji K, Aizawa T, Kiuchi T, Izumi N, et al. Transcriptome profiling reveals infection strategy of an insect maculavirus. DNA Res Int J Rapid Publ Rep Genes Genomes. 2018;25:277. https://doi.org/ 10.1093/dnares/dsx056.

9. Tsukui K, Yagisawa C, Fujimoto S, Ogawa M, Kokusho R, Nozawa M, et al. Infectious Virions of Bombyx Mori latent virus are incorporated into Bombyx Mori Nucleopolyhedrovirus occlusion bodies. Viruses. 2019;11:316. https:// doi.org/10.3390/v11040316.

10. Kawaoka S, Hayashi N, Suzuki Y, Abe H, Sugano S, Tomari Y, et al. The Bombyx ovary-derived cell line endogenously expresses PIWI/PIWIinteracting RNA complexes. RNA. 2009;15:1258-64. https://doi.org/10.1261/ rna.1452209.

11. Geladaki A, Kočevar Britovšek N, Breckels LM, Smith TS, Vennard OL, Mulvey CM, et al. Combining LOPIT with differential ultracentrifugation for highresolution spatial proteomics. Nat Commun. 2019;10:331. https://doi.org/10. 1038/s41467-018-08191-w.

12. Grabherr MG, Haas BJ, Yassour M, Levin JZ, Thompson DA, Amit I, et al. Fulllength transcriptome assembly from RNA-Seq data without a reference genome. Nat Biotechnol. 2011;29:644-52. https://doi.org/10.1038/nbt.1883.

13. Smith-Unna R, Boursnell C, Patro R, Hibberd JM, Kelly S. TransRate: reference-free quality assessment of de novo transcriptome assemblies. Genome Res. 2016;26:1134-44. https://doi.org/10.1101/gr.196469.115.

14. Haas BJ, Papanicolaou A, Yassour M, Grabherr M, Blood PD, Bowden J, et al. De novo transcript sequence reconstruction from RNA-seq using the trinity platform for reference generation and analysis. Nat Protoc. 2013;8:1494-512. https://doi.org/10.1038/nprot.2013.084.

15. Waterhouse RM, Seppey M, Simão FA, Manni M, loannidis P, Klioutchnikov $\mathrm{G}$, et al. BUSCO applications from quality assessments to gene prediction and Phylogenomics. Mol Biol Evol. 2018;35:543-8. https://doi.org/10.1093/ molbev/msx319.

16. Duan J, Li R, Cheng D, Fan W, Zha X, Cheng T, et al. SilkDB v2.0: a platform for silkworm (Bombyx mori ) genome biology. Nucleic Acids Res. 2010;38: D453-6. https://doi.org/10.1093/nar/gkp801.

17. Lu F, Wei Z, Luo Y, Guo H, Zhang G, Xia Q, et al. SilkDB 3.0: visualizing and exploring multiple levels of data for silkworm. Nucleic Acids Res. 2020;48: D749-55. https://doi.org/10.1093/nar/gkz919.

18. Bryant DM, Johnson K, DiTommaso T, Tickle T, Couger MB, Payzin-Dogru D, et al. A tissue-mapped axolotl De novo Transcriptome enables identification of limb regeneration factors. Cell Rep. 2017;18:762-76. https://doi.org/10. 1016/j.celrep.2016.12.063.

19. Abe H, Fujii T, Tanaka N, Yokoyama T, Kakehashi H, Ajimura M, et al. Identification of the female-determining region of the $\mathrm{W}$ chromosome in Bombyxmori. Genetica. 2008;133:269-82. https://doi.org/10.1007/s10709007-9210-1.

20. KATSUMA S, KIUCHI T, KAWAMOTO M, FUJIMOTO T, SAHARA K. Unique sex determination system in the silkworm, Bombyx mori: current status and beyond. Proc Jpn Acad Ser B Phys Biol Sci. 2018;94:205-16. https://doi.org/ 10.2183/pjab.94.014.

21. Scheidler CM, Kick LM, Schneider S. Ribosomal Peptides and Small Protein on the Rise. ChemBioChem. 2019;20:cbic.201800715. https://doi.org/10.1002/ cbic.201800715.
22. Cox J, Mann M. MaxQuant enables high peptide identification rates, individualized p.p.b.-range mass accuracies and proteome-wide protein quantification. Nat Biotechnol. 2008;26:1367-72. https://doi.org/10.1038/nbt. 1511.

23. Song L, Florea L. Rcorrector: efficient and accurate error correction for Illumina RNA-seq reads. GigaScience. 2015:4:48. https://doi.org/10.1186/ s13742-015-0089-y.

24. Martin M. Cutadapt removes adapter sequences from high-throughput sequencing reads. EMBnet J. 2011;17:10. https://doi.org/10.14806/ej.17.1.200.

25. Andrews $S$. FastQC: a quality control tool for high throughput sequence data. Babraham Bioinformatics. 2010; Available: https://www.bioinformatics. babraham.ac.uk/projects/fastqc/. [cited 10 Jul 2019].

26. Dobin A, Davis CA, Schlesinger F, Drenkow J, Zaleski C, Jha S, et al. STAR: ultrafast universal RNA-seq aligner. Bioinformatics. 2013;29:15-21. https://doi. org/10.1093/bioinformatics/bts635.

27. Langmead B, Salzberg SL. Fast gapped-read alignment with bowtie 2. Nat Methods. 2012;9:357-9. https://doi.org/10.1038/nmeth.1923.

28. Camacho C, Coulouris G, Avagyan V, Ma N, Papadopoulos J, Bealer K, et al. BLAST+: architecture and applications. BMC Bioinformatics. 2009;10:421. https://doi.org/10.1186/1471-2105-10-421.

29. Quinlan AR, Hall IM. BEDTools: a flexible suite of utilities for comparing genomic features. Bioinformatics. 2010;26:841-2. https://doi.org/10.1093/ bioinformatics/btq033.

30. R Core Team. R: a language and environment for statistical computing (version 3.5.3) [computer software]. Vienna: R Foundation for statistical computing. R Foundation for statistical Computing; 2019.

31. Eddy SR. Accelerated Profile HMM Searches. Pearson WR, editor. PLoS Comput Biol. 2011;7:e1002195. https://doi.org/10.1371/journal.pcbi.1002195.

32. Almagro Armenteros JJ, Tsirigos KD, Sønderby CK, Petersen TN, Winther O, Brunak S, et al. SignalP 5.0 improves signal peptide predictions using deep neural networks. Nat Biotechnol. 2019;37:420-3. https://doi.org/10.1038/ s41587-019-0036-z

33. Krogh A, Larsson B, von Heijne G, Sonnhammer ELL. Predicting transmembrane protein topology with a hidden markov model: application to complete genomes. J Mol Biol. 2001;305:567-80. https://doi.org/10.1006/ JMBI.2000.4315.

34. Lagesen K, Hallin P, Rødland EA, Stærfeldt H-H, Rognes T, Ussery DW. RNAmmer: consistent and rapid annotation of ribosomal RNA genes. Nucleic Acids Res. 2007;35:3100-8. https://doi.org/10.1093/nar/gkm160.

35. Almagro Armenteros JJ, Sønderby CK, Sønderby SK, Nielsen H, Winther O. DeepLoc: prediction of protein subcellular localization using deep learning. Hancock J, editor. Bioinformatics. 2017;33:3387-95. https://doi.org/10.1093/ bioinformatics/btx431.

36. McKenna A, Hanna M, Banks E, Sivachenko A, Cibulskis K, Kernytsky A, et al. The genome analysis toolkit: a MapReduce framework for analyzing nextgeneration DNA sequencing data. Genome Res. 2010;20:1297-303. https:// doi.org/10.1101/gr.107524.110.

37. Cingolani $P$, Platts $A$, Wang $L L$, Coon $M$, Nguyen $T$, Wang $L$, et al. A program for annotating and predicting the effects of single nucleotide polymorphisms, SnpEff. Fly (Austin). 2012;6:80-92. https://doi.org/10.4161/fly. 19695.

38. Shevchenko A, Tomas H, Havli J, Olsen JV, Mann M. In-gel digestion for mass spectrometric characterization of proteins and proteomes. Nat Protoc. 2006;1:2856-60. https://doi.org/10.1038/nprot.2006.468.

39. Rappsilber J, Mann M, Ishihama Y. Protocol for micro-purification, enrichment, pre-fractionation and storage of peptides for proteomics using StageTips. Nat Protoc. 2007;2:1896-906. https://doi.org/10.1038/nprot.2007. 261.

40. Cox J, Hein MY, Luber CA, Paron I, Nagaraj N, Mann M. Accurate proteomewide label-free quantification by delayed normalization and maximal peptide ratio extraction. Termed MaxLFQ Mol Cell Proteomics. 2014;13: 2513-26. https://doi.org/10.1074/mcp.m113.031591.

41. Perez-Riverol Y, Csordas A, Bai J, Bernal-Llinares M, Hewapathirana S, Kundu DJ, et al. The PRIDE database and related tools and resources in 2019: improving support for quantification data. Nucleic Acids Res. 2019;47:D44250. https://doi.org/10.1093/nar/gky1106.

42. Wehrens R, Kruisselbrink J. Flexible Self-Organizing Maps in kohonen 3.0. J Stat Softw Artic. 2018;87:1-18. https://doi.org/10.18637/jss.v087.i07.

43. Hahsler M, Hornik K. TSP - Infrastructure for the Traveling Salesperson Problem. J Stat Softw. 2007;23:1-21. https://doi.org/10.18637/jss.v023.i02. 
44. Gatto L, Breckels LM, Wieczorek S, Burger T, Lilley KS. Mass-spectrometrybased spatial proteomics data analysis using pRoloc and pRolocdata. Bioinformatics. 2014;30:1322-4. https://doi.org/10.1093/bioinformatics/ btu013.

45. Wu TD, Watanabe CK. GMAP: a genomic mapping and alignment program for mRNA and EST sequences. Bioinformatics. 2005;21:1859-75. https://doi. org/10.1093/bioinformatics/bti310.

\section{Publisher's Note}

Springer Nature remains neutral with regard to jurisdictional claims in published maps and institutional affiliations.

- fast, convenient online submission

- thorough peer review by experienced researchers in your field

- rapid publication on acceptance

- support for research data, including large and complex data types

- gold Open Access which fosters wider collaboration and increased citations

- maximum visibility for your research: over $100 \mathrm{M}$ website views per year

At $\mathrm{BMC}$, research is always in progress. 